Ann. Inst. Statist. Math.

31 (1979), Part A, 349

\title{
CORRECTIONS TO
}

"MULTI-FOLDING THE NORMAL DISTRIBUTION AND MUTUAL TRANSFORMATION BETWEEN UNIFORM AND NORMAL RANDOM VARIABLES"

NAOTO NiKI

(This Annals 31 (1979), 125-140)

\begin{tabular}{lcll}
$\begin{array}{l}\text { Page } \\
129\end{array}$ & Line & \multicolumn{1}{c}{\begin{tabular}{c}
\multicolumn{1}{c}{ Error } \\
{$\left[k(k+2) /(k+1)^{2}\right]$}
\end{tabular}} & {$\left[k(k+2) /(k+1)^{2}\right]^{2 r}$} \\
131 & 15 & $\min$ & $\max$ \\
& 6 & $\frac{2 \exp \left(-\pi^{2} k^{2} /\left(2 a^{2}\right)\right)}{1-\exp \left(-3 \pi^{2} /\left(2 a^{2}\right)\right)}$ & $\frac{2 \exp \left(-\pi^{2} /\left(2 a^{2}\right)\right)}{1-\exp \left(-3 \pi^{2} /\left(2 a^{2}\right)\right)}$ \\
& 8 & $\phi(2 a+x)$ & $2 \phi(2 a+x)$ \\
132 & Fig. 1 & $\phi(2 a-x)$ & $2 \phi(2 a-x)$ \\
& & $\phi(x)$ & $2 \phi(x)$ \\
134 & $3 \uparrow$ & $(K+3)^{2} a^{2} \leqq p^{2}$ & $(K+3)^{2} a^{4} \leqq p^{2}$ \\
137 & 7 & $(2 \mathrm{~b}) x=$ & $(2 \mathrm{~b}) X=$ \\
& 8 & $g_{N}(x) \leqq V$ & $g_{N}(0) \leqq V$
\end{tabular}

The Institute of Statistical Mathematics 